\title{
Censo de aves migratórias (Charadriidae, Scolopacidae e Laridae) na Coroa do Avião, Igarassu, Pernambuco, Brasil
}

\author{
Wallace R. Telino-Júnior ${ }^{1}$, Severino M. de Azevedo-Júnior ${ }^{2} \&$ Rachel M. de Lyra-Neves ${ }^{1}$
}

${ }^{1}$ Departamento de Ecologia e Biologia Evolutiva, Universidade Federal de São Carlos. Endereço para correspondências: Rua 102, número 136, Jardim Paulista, 53407-220 Paulista, Pernambuco, Brasil.E-mail: telinojr@msn.com; rmlneves@msn.com

2 Programa de Pós-graduação em Biologia Animal, Departamento de Zoologia, CCB, Universidade Federal de Pernambuco. Avenida Professor Moraes Rego, 1235, Cidade Universitária, 50670-420 Recife, Pernambuco, Brasil. E-mail: smaj@ufpe.br

\begin{abstract}
Census of shorebirds and Seabirds (Charadriidae, Scolopacidae and Laridae) in the Coroa do Avião, Igarassu, Pernambuco State, Brazil. Thousands of shorebirds and seabirds use the Atlantic route through the northeastern coast of Brazil every year to migrate to South America. In this study was made a census of individuals that uses the Coroa do Avião ( $7^{\circ} 40^{\prime} \mathrm{S}$ and $34^{\circ} 50^{\prime \prime} \mathrm{W}$ ), an islet located on the south bar of the Santa Cruz channel on the north coast of Pernambuco state, from May 1993 throughout April 1995 and from August 1996 throughout July 1997. Four species of the family Charadriidae, seven species of the family Scolopacidae, and two species of the family Laridae was observed. The populational peaks match both the period of arrival to the winter season site and the period of departure to the reproduction site. The species considered very frequent were: Charadrius semipalmatus Bonaparte, 1825 and Calidris alba (Pallas, 1764). All the other species were considered rather frequent. There was a strong linear correlation between 1993 and 1994 among Pluvialis squatarola (Linnaeus, 1758) and Arenaria interpres (Linnaeus, 1758); $A$. interpres and Calidris pusilla (Linnaues, 1766); $A$. interpres and $C$. alba; and C. pusilla and C. alba. From 1994 to April 1995 it occured between P. squatarola and C. alba; C. semipalmatus and C. pusilla; C. semipalmatus and C. pusilla; and A. interpres and C. pusilla. From August 1996 throughout July 1997 there was correlation only between $P$. squatarola and $C$. pusilla. The obtained results demonstrate the great importance of the Coroa do Avião as a winter season site.
\end{abstract}

KEY WORDS. Charadriiformes, direct counting, neartic migrants, terns, waders.

As aves limícolas migratórias chegam à costa brasileira todos os anos em bandos, sendo registradas cerca de 40 espécies pertencentes às famílias Jacanidae, Rostratulidae, Haematopodidae, Charadriidae, Scolopacidae, Recurvirostridae e Burhinidae (AzEVEDO-JÚNIOR 1998) e ainda os da família Laridae, conhecidas por gaivotas e andorinhas-do-mar. Vêem a procura de locais de invernada onde encontram alimentação farta, propiciando a elas a continuidade do seu ciclo de vida.

No Brasil são encontrados vários sítios de invernada, que são de extrema importância para conservação e manutenção destas espécies, desde o Amapá até o Rio Grande do Sul, como a Ilha de Campechá, Maranhão, a Lagoa do Peixe, Rio Grande do Sul e a Coroa do Avião, Pernambuco. Nesta última, milhares de aves das famílias Charadriidae, Scolopacidae e Laridae, se aglomeram todos os anos de agosto a abril, devido à riqueza e disponibilidade de invertebrados marinhos encontrados na ilha durante a baixa-mar (AZEVEDO-JúNIOR et al. 2001b). A fartura de alimento propicia a essas aves a garantia de engorda, dessa forma, adquirem energia suficiente para efetuar as mudas e retornar ao seu sítio de reprodução (AzEvEDo-JúNIOR et al. 2001a, b).

Dentre os Charadriidae e Scolopacidae, 15 espécies são registradas para a área, sendo também encontradas três espécies de gaivotas da família Laridae (AzEvedo-JúNIOR 1992, 1998). Desde 1986 trabalhos envolvendo capturas, marcação, censos e biologia dessas espécies, são realizados na Coroa do Avião. Nesse sentido, esta pesquisa traz novas informações a respeito da população de aves migratórias que utilizam a Coroa do Avião como sítio de alimentação e repouso, no intuito de contribuir com o monitoramento dessas espécies.

\section{MATERIAL E MÉTODOS}

\section{Área de estudo}

A Coroa do Avião é uma formação arenosa recente decorrente do acúmulo de sedimentos trazidos pela deriva litorânea e retidos pela corrente hidráulica formada pelas corrente de fluxo e refluxo que entram e saem na barra do Forte Orange 


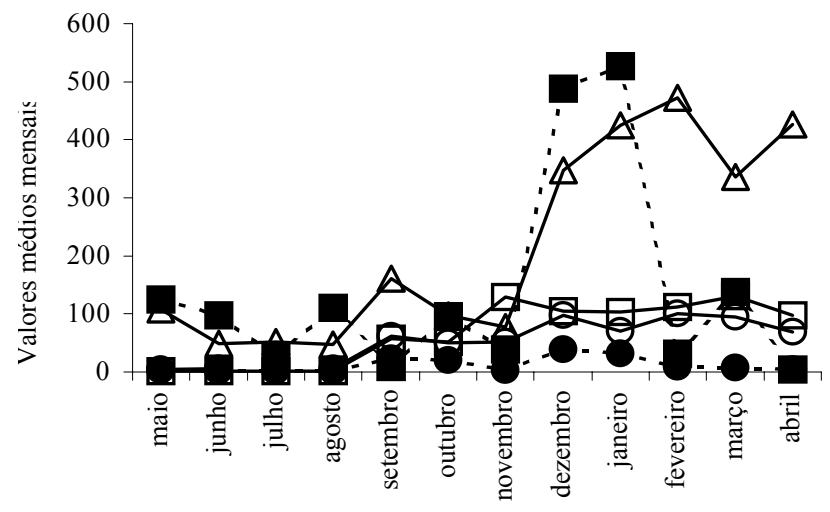

Meses de estudo

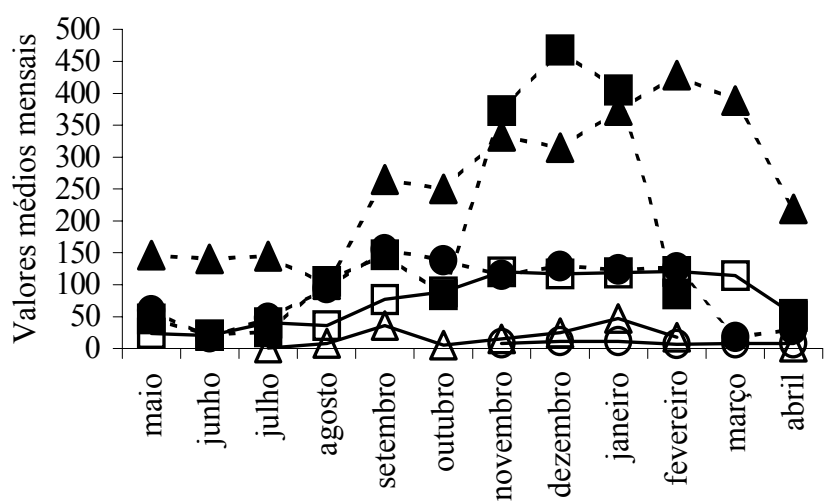

Meses de estudo

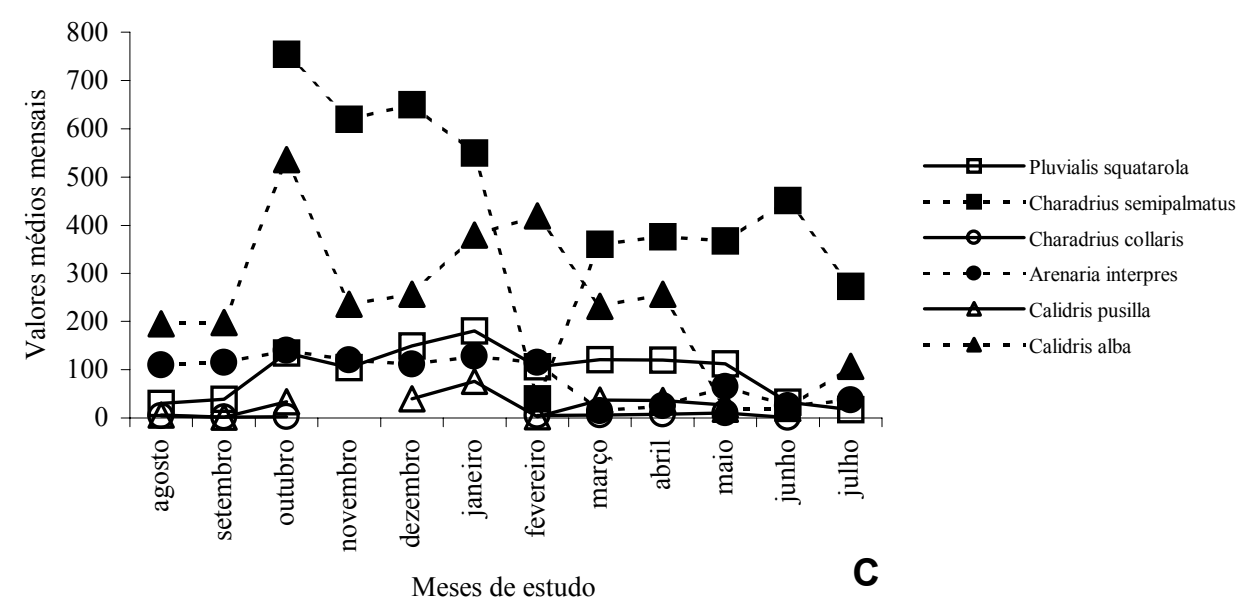

Figura 1. Flutuação populacional de Pluvialis squatarola, Charadrius semipalmatus, Charadrius collaris, Arenaria interpres, Calidris pusilla e Calidris alba na Coroa do Avião entre: (A) 1993 e 1994, (B) 1994 e 1995 e (C) 1996 e 1997.

(AzEvEDo-JúNIOR 1998). Segundo este autor a ilhota localiza-se na barra sul do Canal de Santa Cruz ao norte da praia de Maria Farinha e ao sul da Ilha de Itamaracá sob as coordenadas $7^{\circ} 40^{\prime} \mathrm{S}$ e $34^{\circ} 50^{\prime} \mathrm{W}$. Possui cerca de 2,5 hectares durante a preamar e sua vegetação é característica de restinga com arbustos e gramíneas em expansão. À frente da ilhota, na porção leste, destacam-se os bancos de areia, oriundos dos depósitos de sedimentos. Estes são utilizados como área de pouso das aves migratórias nas preamares. Na porção sul da ilha há um prado de fanerógamas marinhas com grande concentração de invertebrados marinhos que são consumidos por estas aves durante a baixa-mar.

\section{Métodos}

A pesquisa teve duração de 36 meses de coleta, sendo realizada entre maio de 1993 a junho de 1995 e de julho de 1996 a agosto de 1997, com duração de uma semana a cada mês.

Os censos foram realizados nos bancos de areia, em situação de preamar através da contagem direta, segundo a metodologia descrita por Biввy et al. (1992), onde o observador em um ponto fixo desenvolvia contagem individual de cada espécie com auxílio de luneta Seacher ( 20 x 40 x 60 mm), bi- nóculo (10 x $50 \mathrm{~mm})$ e um contador manual eletrônico. As espécies foram identificadas segundo HaRrISON (1983) e HAYMAN et al. (1986) e as citações nas tabelas seguiram a seqüência sistemática de acordo com SicK (1997).

Os dados obtidos foram analisados através da constância, da freqüência, da flutuação populacional e da Correlação de Spearman. Quanto a Constância foi dada através do número de meses contendo a espécie $\boldsymbol{i}$, dividido pelo número total de meses de coleta, sendo: constantes aquelas espécies com percentual acima de 50\%; acessórias, entre $25 \%$ e $50 \%$ e acidentais, abaixo de $25 \%$ (DAJOZ 1983).

A freqüência de ocorrência foi expressa pelo número de indivíduos da espécie $\boldsymbol{i}$ em relação ao total de indivíduos de todas as espécies para cada mês, sendo: muito freqüente (percentual acima de $50 \%$ ); freqüente (entre $25 \%$ a $50 \%$ ) e pouco freqüente (abaixo de 25\%) (AzEvedo-JúnIor \& LaRrazÁbaL 1994).

A flutuação sazonal foi avaliada pelas médias das contagens mensais (AzEVEDo-JúNIOR \& LARRAZÁBAL 1994), para as espécies que foram consideradas constantes.

O coeficiente de correlação de Spearman foi desenvolvido através do programa GraphPad Instat 3.0. Considerou-se 
Tabela I. Constância das espécies limícolas migratórias nos 36 meses de pesquisa, realizada na Coroa do Avião, Igarassu, Pernambuco.

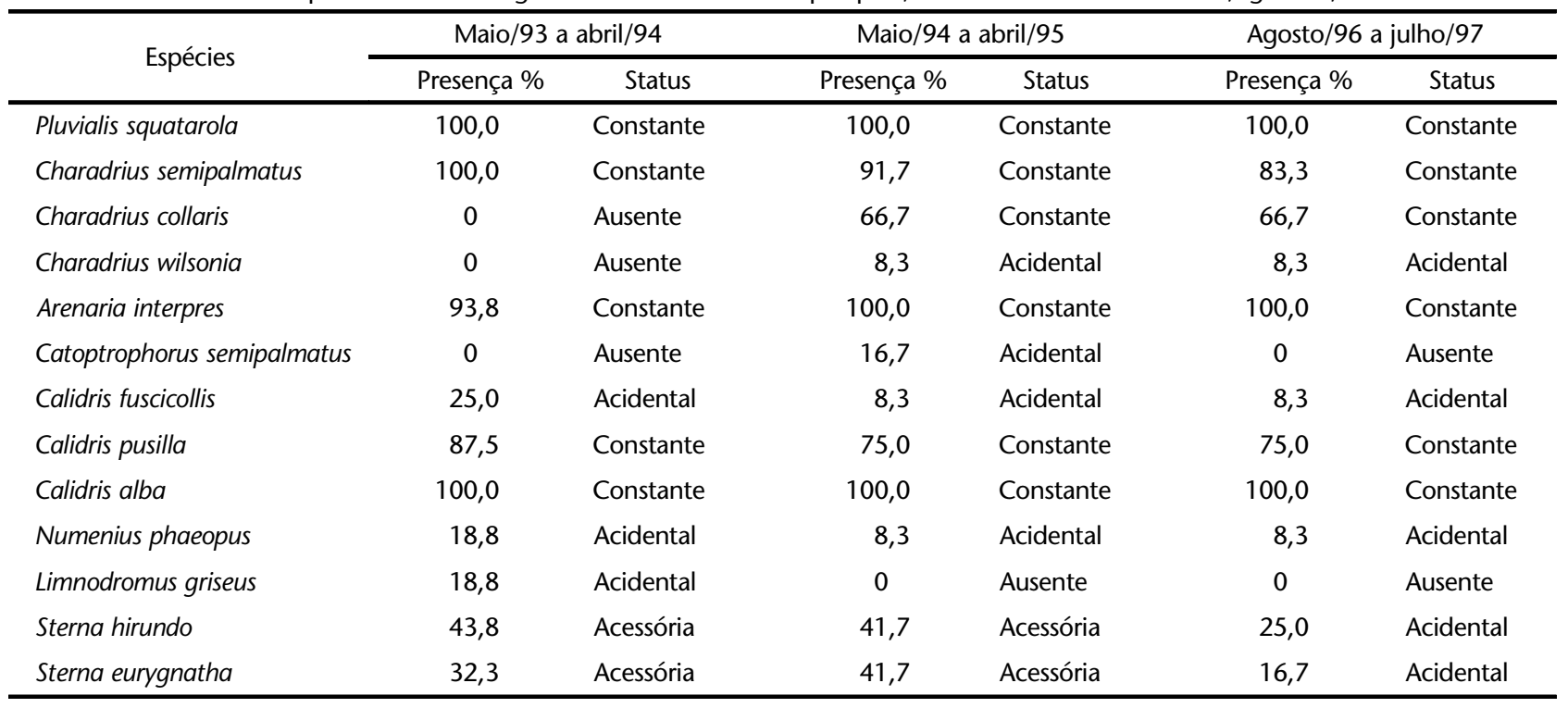

Forte Correlação (FC) os valores obtidos entre 0,70 e 0,89 e Correlação Muito Forte (CMF) para valores entre 0,90 a 1,00 (Fowler E COHEN 1988).

\section{RESULTADOS}

Durante o período de pesquisa foram registradas quatro espécies da família Charadriidae: Pluvialis squatarola, Charadrius semipalmatus, Charadrius collaris Vieillot, 1818 e Charadrius wilsonia Ord, 1814; sete espécies da família Scolopacidae: Arenaria interpres, Catoptrophorus semipalmatus (Gmelin, 1789), Calidris fuscicollis (Vieillot, 1819), Calidris pusilla, Calidris alba, Numenius phaeopus (Linnaeus, 1758) e Limnodromus griseus (Gmelin, 1789); e duas espécies da família Laridae: Sterna hirundo Linnaues, 1758 e Sterna eurygnatha Saunders, 1876.

Foram constantes entre 1993 e 1994 P. squatarola, Charadrius semipalmatus, A. interpres, C. pusilla e C. alba. Nos anos de 1994 a 1995 e de 1996 a 1997, além das espécies anteriormente citadas, também foi constante C. collaris (Tab. I).

Entre os meses de maio de 1993 a abril de 1994, houve pico populacional de $P$. squatarola nos meses de novembro e março; Charadrius semipalmatus em janeiro; A. interpres em dezembro e fevereiro; $C$. pusilla em setembro e dezembro e $C$. alba em setembro, fevereiro e abril (Fig. 1).

No período de maio 1994 a abril 1995 registrou-se picos em novembro e fevereiro para P. squatarola; em setembro e dezembro para Charadrius semipalmatus; em dezembro e janeiro para C. collaris; em setembro e dezembro para A. interpres; em setembro e janeiro para C. pusilla e em setembro, novembro e fevereiro para C. alba (Fig. 1).

Já entre agosto de 1996 e julho 1997, os picos ocorreram nos meses de outubro e janeiro para P. squatarola; outubro, abril e junho para Charadrius semipalmatus; maio para C. collaris; outubro para $A$. interpres; janeiro para $C$. pusilla e outubro e fevereiro para C. alba (Fig. 1).
Nos meses compreendidos entre 1993 a 1994, Charadrius semipalmatus foi muito freqüente nos meses de maio, junho e agosto e freqüente nos meses de julho, outubro, dezembro e janeiro; C. alba foi muito freqüente em julho, fevereiro e abril e freqüente nos demais meses. $P$. squatarola foi freqüente apenas no mês de novembro, as demais espécies foram todas consideradas pouco freqüentes (Tab. II).

No que tange ao período entre maio de 1994 a abril de 1995, como também de agosto de 1996 a julho de 1997, as únicas espécies consideradas muito freqüente e/ou freqüente foram Charadrius semipalmatus e C. alba, tendo as outras, obtido o status de pouco freqüente (Tab. II).

De maio de 1993 e abril de 1994, observou-se uma forte correlação linear (FC) positiva entre as seguintes espécies: $P$. squatarola e $A$. interpres ( $\mathrm{rs}=0.841$ ); $A$. interpres e $C$. pusilla ( $\mathrm{rs}=$ $0.747)$; A. interpres e C. alba (rs $=0.823$ ) e C. pusilla e $C$. alba (rs $=0.709$ ). De maio de 1994 a abril de 1995 a correlação muito forte foi entre $P$. squatarola e $C$. alba (rs = 0.909), Charadrius semipalmatus e C. pusilla (rs = 0.916), e uma forte correlação entre Charadrius semipalmatus e $A$. interpres $(\mathrm{rs}=0.748)$ e $A$. interpres e $C$. pusilla $(\mathrm{rs}=0.796)$. No período entre agosto de 1997 a julho de 1996, foi registrada uma forte correlação, apenas, para $P$. squatarola com C. pusilla ( $\mathrm{rs}=0.880)$.

\section{DISCUSSÃO}

O número de espécies registradas na Coroa do Avião é parcialmente condizente com os citados por AzEVEDO-JúNIOR \& LARRAZÁBAL (1994) entre 1991 a 1992, notou-se diferenças em relação à composição, uma vez que, nesta pesquisa não foram observados indivíduos das espécies: Actitis macularia (Linnaeus, 1766), Calidris canutus (Linnaeus, 1758) e Gelochelidon nilotica (Gmelin, 1789).

Segundo AzEvEdo-JúNIor \& LARRAZÁbAL (1994) foram constantes Pluvialis squatarola e Charadrius semipalmatus, Arenaria 
Tabela II. Freqüência de ocorrência (\%), das espécies registradas para a Coroa do Avião entre os anos de 1993 a 1997. Espécies: Pluvialis squatarola, Charadrius semipalmatus, C. collaris, C. wilsonia, Arenaria interpres, Catoptrophorus semipalmatus, Calidris fuscicollis, C. pusilla, C. alba, Numenius phaeopus, Limnodromus griseus, Sterna hirundo, S. eurygnatha.

\begin{tabular}{|c|c|c|c|c|c|c|c|c|c|c|c|c|c|c|}
\hline Anos & Meses & squatarola & semipalmatus & collaris & wilsonia & interpres & semipalmatus & fuscicollis & pusilla & $a l b a$ & phaeopus & griseus & hirundo & eurygnatha \\
\hline \multirow[t]{12}{*}{1993 a 1994} & mai & 0,40 & 50,20 & - & - & 2,01 & - & 0,40 & 1,21 & 42,97 & - & - & 2,81 & - \\
\hline & jun & 0,61 & 60,12 & - & - & 3,68 & - & - & 1,23 & 30,06 & - & - & 4,30 & - \\
\hline & jul & 1,24 & 33,33 & - & - & - & - & - & 1,24 & 64,19 & - & - & - & - \\
\hline & ago & 0,54 & 59,14 & - & - & 3,22 & - & 3,22 & 0,54 & 25,81 & - & - & 5,38 & 2,15 \\
\hline & set & 15,75 & 2,49 & - & - & 17,13 & - & - & 6,35 & 44,48 & 1,93 & - & 5,52 & 6,35 \\
\hline & out & 14,41 & 27,12 & - & - & 14,12 & - & - & 5,65 & 27,40 & - & - & 3,67 & 7,63 \\
\hline & nov & 43,00 & 13,00 & - & - & 17,00 & - & - & 1,00 & 26,00 & - & - & - & - \\
\hline & dez & 9,72 & 45,19 & - & - & 9,07 & - & - & 3,61 & 32,13 & - & 0,28 & - & - \\
\hline & jan & 8,89 & 45,47 & - & - & 6,04 & - & - & 2,67 & 36,67 & - & 0,26 & - & - \\
\hline & fev & 15,43 & 4,41 & - & - & 13,77 & - & - & 1,10 & 65,01 & - & 0,28 & - & - \\
\hline & mar & 18,36 & 19,49 & - & - & 13,42 & - & - & 0,99 & 47,46 & 0,28 & - & - & - \\
\hline & $\mathrm{abr}$ & 15,83 & 0,65 & - & - & 11,15 & - & 2,58 & 0,81 & 68,98 & - & - & - & - \\
\hline \multirow[t]{12}{*}{1994 a 1995} & mai & 8,63 & 16,55 & - & - & 21,58 & - & 0,72 & - & 52,52 & - & - & - & - \\
\hline & jun & 10,05 & 11,06 & - & - & 8,54 & - & - & - & 70,35 & - & - & - & - \\
\hline & jul & 13,99 & 9,09 & - & - & 16,78 & - & - & 0,35 & 50,70 & - & - & 3,50 & 5,59 \\
\hline & ago & 8,22 & 23,97 & - & - & 21,46 & - & - & 1,83 & 22,83 & 0,46 & - & 12,10 & 9,13 \\
\hline & set & 10,75 & 20,53 & - & 0,14 & 21,65 & - & - & 5,03 & 37,01 & - & - & 0,42 & 4,47 \\
\hline & out & 14,93 & 14,09 & - & - & 23,15 & 1,85 & - & 0,84 & 41,95 & - & - & 0,17 & 3,02 \\
\hline & nov & 12,32 & 38,30 & 0,82 & - & 11,81 & - & - & 1,54 & 34,19 & - & - & 0,10 & 0,92 \\
\hline & dez & 10,94 & 43,78 & 1,03 & - & 12,07 & 0,37 & - & 2,34 & 29,47 & - & - & - & - \\
\hline & jan & 11,03 & 37,53 & 1,02 & - & 11,49 & - & - & 4,36 & 34,57 & - & - & - & - \\
\hline & fev & 15,37 & 10,93 & 0,89 & - & 16,14 & - & - & 2,29 & 54,38 & - & - & - & - \\
\hline & mar & 21,55 & - & - & - & 3,40 & - & - & - & 73,54 & - & - & 1,51 & - \\
\hline & $a b r$ & 14,84 & 14,01 & 2,20 & - & 8,24 & - & - & 0,55 & 60,16 & - & - & - & - \\
\hline \multirow[t]{12}{*}{1996 a 1997} & ago & 7,13 & - & 0,95 & - & 26,13 & - & - & 1,43 & 46,31 & - & - & 8,55 & 9,50 \\
\hline & set & 9,29 & - & 0,48 & - & 27,38 & - & - & 0,48 & 47,14 & 1,90 & - & 0,48 & 12,85 \\
\hline & out & 8,44 & 47,06 & 0,19 & - & 8,74 & - & - & 2,12 & 36,46 & - & - & - & - \\
\hline & nov & 9,59 & 57,20 & - & - & 11,07 & - & - & - & 21,77 & - & - & 0,37 & - \\
\hline & dez & 12,35 & 53,90 & - & - & 9,20 & - & - & 3,32 & 21,23 & - & - & - & - \\
\hline & jan & 13,70 & 41,86 & - & - & 9,74 & - & - & 5,78 & 28,92 & - & - & - & - \\
\hline & fev & 15,41 & 5,81 & 0,73 & - & 16,71 & - & - & 0,44 & 60,90 & - & - & - & - \\
\hline & mar & 15,69 & 46,82 & 0,78 & - & 1,95 & - & - & 4,80 & 29,96 & - & - & - & - \\
\hline & abril & 14,62 & 45,80 & 0,97 & - & 3,05 & - & - & 4,38 & 31,18 & - & - & - & - \\
\hline & mai & 18,67 & 61,33 & 1,67 & - & 10,83 & - & - & 4,50 & 3,00 & - & - & - & - \\
\hline & jun & 6,21 & 84,93 & 0,19 & - & 4,71 & - & 0,19 & - & 3,77 & - & - & - & - \\
\hline & jul & 3,90 & 62,61 & - & 0,46 & 8,49 & - & - & - & 24,54 & - & - & - & - \\
\hline
\end{tabular}

Revista Brasileira de Zoologia 20 (3): 451-456, setembro 2003 
interpres e Calidris alba e Sterna hirundo. Neste estudo, além das espécies anteriormente citadas, também, foram constantes, Charadrius collaris e Calidris pusilla. Entretanto, as espécies da família Laridae foram consideradas acessórias.

Os picos populacionais analisados coincidem com os períodos de chegada aos trópicos e de partida para o Hemisfério Norte. O aumento da concentração de indivíduos durante a migração, também é citado por AzEVEDO-JÚNIOR \& LARRAZÁBAL (1994) na Coroa do Avião em anos anteriores, sendo, também, comprovados em outra área de invernada no Rio Grande do Norte (LarrazÁbal et al. 2002).

O alto número de indivíduos de C. semipalmatus e C. alba nos meses de junho e julho, período em que estas aves deveriam estar nas suas áreas de reprodução, é comentado por AzeveDo-JúNIOR \& LaRRAZÁbal (1994) e AzEVEDO-JúNIOR et al. (2001a, b). Estes fazem referência à permanência de jovens, sub-adultos e adultos, que não concluem as mudas das penas de vôo, em seu sítio de invernada aguardando o próximo período reprodutivo.

Nos três anos de estudo na Coroa do Avião, o status de muito freqüente, foi atribuído para C. semipalmatus e C. alba. Segundo Azevedo-Júnior \& LarrazÁbal (1994), a única espécie considerada muito freqüente para a área, durante 1991 a 1992 , foi Calidris alba. O aumento do número de indivíduos registrados nos censos, para C. semipalmatus, entre 1993 e 1997 explica este resultado.

Segundo Parker et al. (1996), Numenius phaeopus apresenta uma alta sensibilidade a distúrbios ambientais. Apesar desta espécie de ter sido considerada acidental, sua população aumentou na Coroa do Avião entre 1993 a 1997. AzEvEDo-JúNIOR \& LARRAZÁbAl (1994), registraram um exemplar desta espécie entre 1991 e 1992, período em que existia o uso indiscriminado de aviões ultraleves, os quais perturbavam o pouso e a forragem dos maçaricos Azevedo-JúnIor \& LarRAZÁbal (1997). Ainda de acordo com os mesmos autores, foram tomadas, posteriormente, medidas impedindo o pouso e sobrevôo das aeronaves na ilhota, propiciando o retorno de espécies consideradas sensíveis a este tipo de impacto negativo.

A correlação de Spearman demonstra uma forte dependência na migração destas espécies entre os anos de 1993 a 1995. De 1996 a 1997 é evidenciada a correlação apenas para duas espécies. De acordo com Sick (1983), Morrison (1984), MorRISON \& Ross (1989) e LARRAZÁ́bAl et al. (2002) as aves neárticas se movimentam de acordo com a proximidade do inverno boreal.

A fidelidade destas aves à Coroa do Avião, observada nesta pesquisa e em outras (e.g. AzEVEDo-JúNIOR \& LARRAZÁbAL 1994), indica esta ilhota como sítio de invernada, reforçando sua importância para conservação dos maçaricos e gaivotas que utilizam a área. Segundo Castro \& Myers (1987) e Azevedo-Júnior et al. (2002), espécies migratórias são fiéis aos seus sítios de invernada em suas movimentações sazonais.

Um monitoramento constante deve ser realizado anualmente para acompanhar a variação populacional dessas aves, só assim será possível avaliar declínios e aumentos ocorridos ao longo dos anos e inferir qual a decorrência deste fenômeno. Segundo CoRdeiro et al. (1996) a conservação de aves migratórias está diretamente relacionada com a identificação de sítios de alimentação, repouso e reprodução, e que a perda de um sítio pode acarretar na diminuição e até mesmo na extinção de alguma espécie. Ainda defendem que trabalhos de monitoramento de populações migratórias são fundamentais para conservação das mesmas.

\section{AGRADECIMENTOS}

Agradecemos a Pró-Reitoria de Extensão e de Campi Avançados da Universidade Federal Rural de Pernambuco responsáveis pela administração da Coroa do Avião durante o período de pesquisa. Aos referees da RBZ pelas sugestões pertinentes. Ao Programa Institucional de Bolsas de Iniciação Cientifica (Pibic/ Cnpq) pela bolsa concedida a W. R. Telino-Júnior no período de agosto de 1994 a julho de 1995.

\section{REFERÊNCIAS BIBLIOGRÁFICAS}

Azevedo-JúnIOR, S.M. DE. 1992. Anilhamento de aves migratórias na Coroa do Avião, Igarassu, Pernambuco, Brasil. Caderno Ômega da Universidade Federal Rural de Pernambuco, Série Ciências Aquáticas, Recife, 3: 31-47.

. 1998. As aves do canal de Santa Cruz, Pernambuco, Brasil. Caderno Ômega da Universidade Federal Rural de Pernambuco, Série Ciências Aquáticas, Recife, 5: 35-50.

Azevedo-Júnior, S.M. de \& M.E. LarrazÁbal. 1994. Censo de aves limícolas na Coroa do Avião, Pernambuco, Brasil, informações de 1991 a 1992. Revista Nordestina de Zoologia, Recife, 1 (1): 263-277.

. 1997. Uma proposta de legislação para a conservação das aves limícolas na Coroa do Avião, Pernambuco, Brasil.. Ararajuba, Belo Horizonte, 5 (1): 63-65.

Azevedo-Júnior, S.M. de; M.M. Dias \& M.E. Larrazábal. 2001a. Plumagens e mudas de Charadriiformes (Aves) no litoral de Pernambuco, Brasil. Revista Brasileira de Zoologia, Curitiba, 18 (3): 657- 672.

Azevedo-Júnior, S. M. DE; M.M. Dias; M.E. Larrazábal; W.R. TelinoJúnior; R.M. LyRa-Neves \& C.J.G. Fernandes. 2001b. Recapturas e recuperações de aves migratórias no litoral de Pernambuco, Brasil. Ararajuba, Brasília, 9 (1): 33-42.

Azevedo-Júnior, S.M. de; M.M. Dias; M.E. Larrazábal \& C.J.G. FERnANDes. 2002. Capacidade de vôo de quatro espécies de Charadriformes (Aves) capturadas em Pernambuco, Brasil. Revista Brasileira de Zoologia, Curitiba, 19 (1): 183- 189.

Bıввy, C.J.; N.D. Burgess \& D.A. Hill. 1992. Birds census techniques. London, Academic Press Inc., 257p.

CASTRO, G. \& J. P. Myers. 1987. Ecología y conservación del playero blanco (Calidris alba) en el Peru. Bol. Lima, Lima, 52:47-61.

Cordeiro, P.H. C.; J.M. Flores \& J.L.X. do Nascimento. 1996. Análise das recuperações de Sterna hirundo no Brasil entre 1980 e 1994. Ararajuba, Belo Horizonte, 4: 3-7.

DAJOz, R. 1983. Ecologia Geral. Petrópolis, Vozes, IV+472p.

Fowler, J. \& L. Cohen. 1988. Statistics for ornithologists. Leicester, British Trust for Ornithology, II+150p.

HaRrison, P. 1983. Seabirds, an identification guide. Boston, Houghton Mifflin Co., 448p.

Hayman, P.; J. Marchant \& T. Prater. 1986. Shorebirds: an identification guide. Boston, Houghton Mifflin Co., 412p.

Larrazábal, M.E. de; S. M. de Azevedo-Júnior \& O. Pena. 2002. Monitoramento de aves limícolas na Salina Diamante Branco, Galinhos, Rio Grande do Norte, Brasil. Revista Brasileira de Zoologia, Curitiba, 19 (4): 1081-1089.

Morrison, R.I.G. 1984. Migration systems of some new world shorebirds, p. 125-202. In: J. Burger \& B.L. Olla (Eds). Behavior of marine animals. Shorebirds: migration and 
forangins behavior. New York, Plenum Press, VI+743p. MorRison, R.I.G. \& R.K. Ross. 1989. Atlas of neartic shorebirds on the coast of South America. Ottawa, Canadian Wildlife Service, I+128p.

Parker III, T.A.; D.F. Stotz \& J.W. Fitzpatrick. 1996. Ecological and distributional databases, p. 113-436. In: D.F. SтотZ; J.W. FitzPatrick; T A. Parker III \& D.K. Moskovits (Eds). Neotropical birds: ecology and conservation. Chicago, University of Chicago Press, XI+700p.

SICK, H. 1983. Migração de aves na América do Sul continental. Brasília, Instituto Brasileiro de Desenvolvimento Florestal, II+86p.

1997. Ornitologia brasileira. Rio de Janeiro, Nova Fronteira, 862p.

Recebido em 06.III.2003; aceito em 14.VIII.2003.

Revista Brasileira de Zoologia 20 (3): 451-456, setembro 2003 